\title{
The Evaluation of Acute Pancreatitis into Acute Recurrent Pancreatitis in Children: The Impact of Etiological Factors
}

\author{
Anna Kofla-Dlubacz, M.D., Ph.D., Tatiana Jamer M.D., Katarzyna Akutko * M.D., Ph.D., \\ Tomasz Pytrus M.D., Ph.D., Barbara Iwańczak M.D., Ph.D., Prof. Andrzej Stawarski M.D., Ph.D. \\ $2^{\text {nd }}$ Department of Paediatrics, Gastroenterology and Nutrition, Wroclaw Medical University, Poland \\ *Corresponding author: Katarzyna Akutko, M.D., Ph.D.; katarzyna.akutko@umw.edu.pl
}

Received 24 October 2021;

Accepted 08 November 2021;

Published 10 November 2021

\begin{abstract}
Background: The incidence of acute pancreatitis (AP), acute recurrent pancreatitis (ARP) and chronic pancreatitis (CP) in pediatric population is rising and becomes common clinical problem in gastroenterology. The assessment of morbidity risk factors is necessary to improve the quality of diagnosis, to determine further treatment and to identify groups with poor prognosis of the evolution of AP into ARP and CP. Objectives: The assessment of etiological factors in acute and acute recurrent pancreatitis in children. Material and methods: From January 2015 to November 2017, data on etiological factors responsible for the onset of AP and ARP were collected in a group of pediatric patients hospitalized at the Department of Paediatrics, Gastroenterology and Nutrition, Medical University of Wroclaw, Poland. The study group consisted of 39 patients (27 patients with AP, 11 patients with ARP and 1 with CP). Results: The potential etiological factor was established in $85.2 \%$ of children with AP and in $100 \%$ of children with ARP. Mutations in the SPINK1 gene were found in $44.4 \%$ of children from the ARP group, no mutations in the PRSS1 gene were found in this group. In all patients with a genetic predisposition (SPINK1 mutation) during the first episode of the ARP, coexistence of an additional predisposing factor was found. Conclusions: Patients with ARP require diagnostics towards mutations predisposing to the transition of AP into a chronic disease. The interaction of additional triggers plays a role in the development of pancreatitic inflammatory disease in genetically predisposed individuals.
\end{abstract}

Keywords: pancreatitis, children, epidemiology, genetic predisposition

\section{Introduction}

In recent years, there has been an increase in the recognition of pancreatic diseases in children ${ }^{[1]}$. Acute pancreatitis (AP), acute recurent pancreatitis (ARP) and chronic pancreatitis (CP) becomes a common clinical problem and be difficult for practitioners due to the potentially severe, life-threatening course of the disease. In 2012 the international INSPPIRE working group published a report systematizing the state of the current knowledge on the mentioned subject, specifying the definition of AP, ARP and $\mathrm{CP}$, diagnostic criteria as well as presenting data on the course, treatment and etiology of pancreatic diseases ${ }^{[2,3]}$. For the diagnosis of acute pancreatitis, two out of the following three criteria must be met: clinical symptoms, increase in pancreatic enzymes: lipase and / or amylase in the plasma 3 times above the upper reference limit and / or typical changes of pancreatits in imaging tests. Recurrent acute pancreatitis is diagnosed in patients who have had more than two episodes of AP, in whom laboratory normalization occurs between episodes. In the following years, further publications expanding the knowledge on pancreatic inflammatory diseases in children have been published ${ }^{[4,5]}$. Estimation of the factors involved in etiology of pediatric pancreatitis seems to be extremely important with specific role of genetic predispositions responsible for the early appearance of the disease ${ }^{[6]}$. The mechanism leading to the development of symptoms of acute pancreatitis is associated with mutations in the genes regulating the physiological activation of trypsinogen. The mutation of the $\mathrm{pR} 122 \mathrm{H}$ serine protease in the PRSS1 gene coding for the trypsinogen, leads to excessive selfactivation of the trypsinogen to the trypsin within the pancreatic parenchyma. Under physiological conditions, the increased activation of trypsinogen is prevented by parallel secretion of a serine protease inhibitor, encoded by the SPINK1 gene, which neutralizes about $13 \%$ of trypsin ${ }^{[7]}$. This is a temporary neutralization that prevents premature activity, but does not affect the total content of trypsin in the pancreatic juice and its target function. Another defensive mechanism is chymotrypsin C responsible for the degradation of active trypsin. The efficiency of the described mechanisms determines the proper functioning of the pancreas. Mutations causing their dysregulation, involved in 
trypsinogen hyperactivation (PRSS1), impaired inhibition of trypsinogen self-activation (SPINK1) or active trypsin degradation (loss of CTRC function) lead to an increased risk of developing acute pancreatitis passing into acute recurrent and chronic pancreatitis ${ }^{[7]}$. Coexistence of several mutations increases the risk of chronic pancreatic disease ${ }^{[8]}$. However, genetic mutations are found only in some children developing pancreatitis (5-8\%). The impact of acquired factors in AP, ARP and CP is also important ${ }^{[1]}$. It has been estimated that abdominal trauma, classically associated with the onset of AP, ranges from 10 to $40 \%{ }^{[1]}$. The pathology of the bile ducts and pancreatic ducts (the pancreas divisum, the Oddi sphincteric pathology, cholelithiasis) is described in 10 to $30 \%$ of cases. The infective agent during an episode of acute pancreatitis is found in about $10 \%$ of patients. Mycoplasma pneumoniae, coxackie viruses ${ }^{[1]}$ have been mentioned as commonly engaged in the occurrence of AP but the involvment of other infections should be taken into consideration. It is worth mentionioning that it is frequent that extended diagnosis is not done during the first episode of AP as the patients are hospitalised in the basic medical health care and no precise data are available. The impact of infection often requires differentiation with the response to medication used in therapy, as drugs may cause acute pancreatitis in up to $25 \%$ of patients ${ }^{[1]}$. Among the drugs that potentially induce AP are valproic acid, azathioprine, and 6-mercaptopurine but also antibiotics may play a role in pancreatitis. Also in this case, the difficulty in interpretation is caused by the drug- AP relationship, and the condition that causes the drugs to be taken, in particular autoimmune diseases (systemic lupus erythematous, inflammatory bowel disease). Further analysis of the causative agents seems to be justified.

\section{Objectives}

Retrospective analysis of the etiology in pediatric pancreatitis (AP, ARP, CP).

\section{Materials and methods}

The study comprised 39 patients hospitalized in the II Clinic and Department of Pediatrics, Gastroenterology and Nutrition of the Medical University of Wroclaw in 2015-2017. 27 patients in this period were diagnosed with acute pancreatitis (AP), 11 acute recurrent pancreatitis (ARP) and 1 with chronic pancreatitis (CP). Diagnosis of AP was based on clinical symptoms and laboratory parameters -> 3-fold increase in reference values of pancreatic enzyme activity - amylase and / or lipase. There were 20 boys and 19 girls in the study group.

The average age at the disease onset (episode of $\mathrm{AP} /$ the first episode of ARP, first symptoms of CP) was 10 years and 9 months. The youngest patient at the time of diagnosis was 2 years and 5 months, the oldest was 17 years and 6 months. The data from medical history, the results of laboratory and imaging tests were analyzed. Distinction for the congenital and acquired factors was done. The frequency of the mutations in genes SPINK1 and PRSS1 predisposing to AP and ARP was determined. Exon 3 in the SPINK1 gene and exons 2, 3 in the PRSS1 were examined. The effects of infectious agents were analyzed - for this purpose the results of serological tests for selected infections were reviewed (coxackie A7, B7- IgM, IgG by Elisa, mycoplasma pneumoniaeIgM, IgG by ELISA, CMV, EBV, - IgM, IgG against the capsid and nuclear antigen). The data from the patient's history were also analyzed regarding the symptoms / diagnosis of infection at the time of AP / ARP, before hospitalization. Among other diagnostic tests performed in patients, the results of the test for mutations in the CFTR gene and the results of pilocarpine iontophoresis were analyzed. In the CFTR gene exon 9, 10, 11 were tested by DNA sequencing. The level of IgG4 antibodies, the increase of which is observed in autoimmune pancreatitis type one was checked. The results of imaging examinations were analyzed: magnetic resonance imaging (MRI), MRCP, abdominal computed tomography (CT), ultrasound examination of the abdominal cavity (USG), in search of the anatomical cause of development of AP, ARP. Data about abdominal trauma and the involvment of other exogenous factors, including the use of drugs were colected. The tests performed in patients differed depending on the clinical indications. The results are presented in a descriptive form. The analysis was retrospective.

\section{Results}

Genetic testing for mutations in the SPINK1 and PRSS1 genes was performed in 8 children from the group of 27 patients with AP (first group) and 9 children from the group of 11 patients with ARP (second group). Among patients with a single episode of acute pancreatitis, the patients with severe course of AP were qualified for genetic testing. There was no mutation within the SPINK1 gene in this group. In one patient with acute pancreatitis, mutation in the PRSS1 gene was detected - c.365G>ApR122H. Patient's family history was positive with diseases of the pancreas (father of $\mathrm{CP}$, grandmother on the father's side pancreatic cancer). In this child during the episode of AP we identified the serologic markers of the EBV seroconversion. During two years observation no relapse was observed. In the second group - ARP, in 4 out of 9 patients (44.4\%), a mutation in exon 3 of the SPINK1 gene - c. 1001A> G pN34S, was detected. Among all 4 patients with ARP and confirmed mutation in the SPINK1 gene, an additional factor triggering the occurrence of the first ARP episode was established, in 3 it was serologically confirmed infection with the coxackie virus (in two A7, B7, in one B7). In the fourth patient, the first episode of the ARP was preceded by an abdominal injury. None of the patients with ARP showed mutations in exons 2 and 3 in the PRSS1 gene. In two children who did not undergo genetic testing, biliary ARP was confirmed (biliary cholelithiasis, common bile duct stenosis).

The infectious agent was established in serological tests and / or data from the interview in 11 patients from the first- AP group $(40.7 \%)$. In two elevated IgM antibodies against coxackie virus (A7, B7) were found. In one of the patients immediately before the onset of AP and befor hospitalisation, pharyngitis was diagnosed. In serological examinations performed during the AP, but after symptomatic infection of the throat, positive $\mathrm{IgG}$ antibodies against coxackie A7, B7 were detected. Among the other patients, one showed positive IgM antibodizes against chlamydia pneumoniae, two against mycoplasma pneumoniae infection and the second two symptoms of AP developed during EBV infection (one patient had positive IgM antibodies and the next seroconversion of EBV infection). One of the patients developed AP in the course of chickenpox. One patient was treated with the first generation cephalosporin due to throat infection with fever, another patient had acute pancreatitis during the first phase of Lyme disease (infection confirmed by the ELISA method, verified by Western blotting). In the second group - the ARP, in addition to three patients with confirmed coxackie virus infection and a co-existing SPINK1 mutation, one patient was diagnosed with mycoplasma pneumonia (IgM) infection during the first episode. The described patient was hospitalized twice in the clinic; 
during the second stay the symptoms of acute pancreatitis were preceded by alcohol consumption. Patients with confirmed infectious agent during the first ARP episode constituted $36.3 \%$ of all patients.

Pilocarpine iontophoresis was performed in 10 children from the first group - AP and 7 from the second group - ARP. In the first group, the test result in all patients was negative. In the second group, two patients had an abnormal pilocarpine iontophoresis result. In patients with abnormal results of iontophoresis, a genetic test was performed. In one case, the presence of a single P.Phe508del CFTR mutation (heterozygote) was found and the patient was referred for further diagnosis towards rare mutations, without finally confirming the diagnosis, the child is under observation. In the second patient c.1210-12T mutation was detected (9-year-old patient, healthy before ARP), verification of polycarpine iontophoresis did not confirm positive result, however, in this child the SPINK1 mutation coexisted - c. $101 \mathrm{~A}>\mathrm{G}$ pN34S. Heterozygous mutation in the CFTR-c 1210-12T gene, with the correct result of pilocarpine iontophoresis, was found in two patients from the group 1 (AP).

Elevated IgG4 levels were found in one patient with ARP. The girl's family history was positive with diseases of the pancreas, her mother had autoimmune pancreatitis, maternal grandmothers - pancreatic cancer. In the study group one patient developed chronic pancreatitis es an extraintestinal manifestation of inflammatory bowel disease, with no elevation in IgG4 level.

In the first group -AP, in one child (3.7\%), symptoms of the disease occurred as a complication of the endoscopic examination of the upper gastrointestinal tract (intramural hematoma of the duodenum). Symptoms of AP resolved in the course of hematoma involution. In three $(11.1 \%)$ children AP occurred immediately after abdominal injury.

In three children (8.8\% from the group AP + ARP) a relationship between the symptoms of pancreatitis and valproic acid intake was established (two children with AP and one of the $\mathrm{ARP}$ - triggering factor of the first episode); in the next four AP occurred with the administration of azathioprine in the course of inflammatory bowel disease.

In the group of children with ARP, the pathology of bile and / or pancreatic duct occurred in three children (27.3\%). In one boy pancreas divisum was diagnosed, in two children pathologies of the common bile duct (narrowing of the common bile duct, choledocholithiasis). Abnormalities of the common bile duct (stenosis) were found in one case in the group of children with AP $(3.7 \%)$, no relapses were observed in the time of study. Among the studied children, the etiological factor was not determined in five children with acute pancreatitis $(17.8 \%)$. The share of individual etiological factors in the development of AP and ARP is presented in Table 1 and 2.

Table 1: Etiological factors of acute pancreatitis

\begin{tabular}{|l|l|l|l|}
\hline Etiological factors of acute pancreatitis (AP) in study group & $\begin{array}{l}\text { Number of tested patients/ } \\
\text { Number of diagnosed AP } \\
\text { patients }\end{array}$ & $\begin{array}{l}\text { Number of patients with } \\
\text { confirmed etiologic factor/ } \\
\text { Number of tested patients }\end{array}$ & $\begin{array}{l}\text { Number of patients with confirmed } \\
\text { etiologic factor- specification/ Number of } \\
\text { patients with confirmed etiologic factor }\end{array}$ \\
\hline Postulated etiological factor & $8 / 27$ & $1 / 8$ & PRSS1 mutation \\
\hline $\begin{array}{l}\text { Genetic predisposition } \\
\text { (SPINK1/PRSS1 mutations) }\end{array}$ & $27 / 27$ & $11 / 27$ & $\begin{array}{l}3 / 11 \text { coxackie virus } \\
1 / 11 \text { chlamydia pneumoniae } \\
2 / 11 \text { mycoplasma pneumoniae } \\
2 / 11 \text { EBV } \\
1 / 11 \text { chickenpox } \\
1 / 11 \text { Lyme disease } \\
1 / 11 \text { infection of the throat with fever }\end{array}$ \\
\hline Infectious agent & & & \\
\hline Abdominal trauma & & $3 / 27$ & $\begin{array}{l}2 / 6 \text { - valpronic acid } \\
4 / 6 \text { azathioprine }\end{array}$ \\
\hline Pharmacotherapy & $27 / 27$ & $6 / 27$ & One episode during observation \\
\hline Obstructive agent & $27 / 27$ & $1 / 27$ & Intramural hematoma of the duodenum \\
\hline Complication of gastroscopy & & $1 / 27$ & \\
\hline
\end{tabular}

Table 2: Etiological factors of acute recurrent pancreatitis

\begin{tabular}{|c|c|c|c|c|}
\hline \multicolumn{5}{|c|}{ Etiological factors of acute reccurrent pancreatitis (ARP) in study group } \\
\hline Postulated etiological factor & $\begin{array}{l}\text { Number of tested } \\
\text { patients/ Number of } \\
\text { diagnosed ARP } \\
\text { patients }\end{array}$ & $\begin{array}{l}\text { Number of patients with } \\
\text { confirmed etiologic } \\
\text { factor/ Number of tested } \\
\text { patients }\end{array}$ & $\begin{array}{l}\text { Number of patients with } \\
\text { confirmed etiologic factor- } \\
\text { specification/ Number of } \\
\text { patients with confirmed } \\
\text { etiologic factor }\end{array}$ & Notes \\
\hline $\begin{array}{l}\text { Genetic predisposition } \\
\text { (SPINK1/PRSS1 mutations) }\end{array}$ & $9 / 11$ & $4 / 9$ & 4/4 SPINK1 mutation & $\begin{array}{l}\text { 3/4 SPINK1 mutation+ } \\
\text { infection } \\
\text { (1/3+ CFTR mutation) }\end{array}$ \\
\hline Infectious agent & $11 / 11$ & $4 / 11$ & $\begin{array}{l}3 / 4 \text { coxackie virus } \\
1 / 4 \text { mycoplasma pneumoniae }\end{array}$ & $\begin{array}{l}\text { 1/3 infection+ } \\
\text { SPINK1mutation }\end{array}$ \\
\hline $\begin{array}{l}\text { Elevation of serum IgG4 } \\
\text { level }\end{array}$ & $11 / 11$ & $1 / 11$ & & \\
\hline Pharmacotherapy & $11 / 11$ & $1 / 11$ & valpronic acid & \\
\hline Obstructive agent & $11 / 11$ & $2 / 11$ & $\begin{array}{l}1 / 2 \text { cholelithiasia } \\
1 / 2 \text { pancreas divisum }\end{array}$ & \\
\hline
\end{tabular}




\section{Discussion}

Mutations in the SPINK1 (serine protease inhibitor Kazal type 1) and PRSS1 (cationic tripsinogen) genes as well as pathologies of pancreatic ducts are indicated as the main causes responsible for the evolution of acute pancreatitis into acute recurrent and chronic pancreatitis ${ }^{[9]}$. In the studies of Kumar et al. ${ }^{[8]}$, the SPINK 1 N34S mutation has been found in $25 \%$ of patients with ARP and CP, which differed significantly from the population of healthy children, where the mutation frequency is estimated at $1-3 \%$. Our research revealed the presence of mutations in the SPINK1 gene $\mathrm{N} 34 \mathrm{~S}$ in $44.4 \%$ of children with acute recurrent pancreatitis, while no mutations in the PRSS1 gene were found in any ARP patients. No SPINK1 mutation was detected in any patient in the group of children with a single AP episode. These results confirm that genetic factors predominantly determine the development of chronic pancreatic disease which is compatible with the literature data ${ }^{[7,10,11]}$. It is noteworthy that among patients with ARP and mutations in the SPINK1 gene, during the first episode of the ARP, there was an additional triggering factor like as infection and / or abdominal injury.

In one of the patients several factors predisposing to the development of the ARP coexisted, SPINK1 mutation, mutation in the CFTR gene, abdominal trauma and coxackie virus infection. The available literature indicates the participation of several factors that, while acting together, affect the activation of the extensive trypsin- dependent pathway of acute pancreatitis. Genetic predispositions can facilitate premature activation, insuffitient inhibition and / or degradation of trypsin, which, while impeding the outflow of pancreatic juice, leads to the development of inflammation in the pancreas parenchyma. INSPIRRE data show that $9.5 \%$ of patients with ARP reveled the presence of two genetic mutations. The most common combination was: SPINK1 / CFTR. ${ }^{[8]}$ as well as pathologies in pancreatic ducts are indicated as the main causes responsible for the evolution of acute pancreatitis in acute recurrent and chronic pancreatitis ${ }^{[9]}$. In the studies of Kumar et al. ${ }^{[8]}$, the SPINK $1 \mathrm{~N} 34 \mathrm{~S}$ mutation was found in $25 \%$ of patients with ARP and $\mathrm{CP}$, which was a significant difference to the population of healthy children, where the mutation frequency is estimated at $1-3 \%$. Our research revealed the presence of mutations in the SPINK1 gene N34S in $44.4 \%$ of children with acute recurrent pancreatitis, while no mutations in the PRSS1 gene were found in any ARP patient. No SPINK1 mutation was detected in any patient in the group of children with a single AP episode.

These results confirm that genetic factors predominantly determine the development of chronic pancreatic disease which is consistent with the literature data ${ }^{[7,10,11]}$. It is noteworthy that among patients with ARP and mutations in the SPINK1 gene, during the first episode of the ARP, there was an additional triggering factor in the form of infection and / or abdominal injury. One of the patients coexisted with several factors predisposing to the development of the ARP, mutation SPINK1, mutation in the CFTR gene, abdominal trauma and coxackie virus infection. The available literature indicates the participation of several factors that, while acting together, affect the activation of trypsindependent acute pancreatitis. Genetic predisposition is conditioned by premature activation, disorders of inhibition and / or degradation of trypsin, which, while impeding the outflow of pancreatic juice, lead to the development of inflammation in the pancreas. By INSPIRE date $9.5 \%$ of patients with ARP showed the presence of two genetic mutations, with the most common combination: SPINK1 / CFTR ${ }^{[8]}$.
According to Schneider et al. CFTR mutation p.R75q, responsible for the defect of trans membrane transport of bicarbonates in people with preserved function of trans membrane transport of chlorides (without confirmed cystic fibrosis) impairs the secretory function of the pancreas leading to intra-parenchymal activation of trypsin in $\mathrm{AP}^{[12]}$.

The coexistence of the SPINK1 and CFTR mutations increased 900 times the risk of developing chronic disease of pancreas (ARP and CP) ${ }^{[8]}$. However, these studies require extension, because the role of mutations in the CFTR gene has been questioned by Rosendahl et al. - only CFTR mutations associated with cystic fibrosis increased statistically significantly the risk of developing chronic pancreatitis. Studies on a group of 660 patients with CP have not shown an increased incidence of mutations in the CFTR gene, which were not associated with cystic fibrosis compared to the control group ${ }^{[10]}$. The pathology of the biliary and pancreatic ducts play an important role in the development of AP ${ }^{[1,13]}$. In our studies, it occurred in $27.3 \%$ of patients with ARP and one patient with a single AP episode (3.6\%) which corroborates with INSPIRRE data, according to which the obstructive cause in pancreatitis (AP and ARP) is estimated at 33\% of patients ${ }^{[8,14]}$. In cases of biliary pancreatitis, the issue to consider is surgical or endoscopic intervention ${ }^{[14,15]}$.

However, it requires an individual approach, due to the specificity of the pediatric population, and the decision on the technique of intervention is often very problematic. The pancreas divisum, which is a known predisposing factor for the occurrence of AP and ARP, may indirectly, through accompanying anomalies in the bile ducts, lead to AP/ARP. It should be mentioned that $95 \%$ of patients with pancreas divisum do not show any symptoms of pancreatitis in their lifetime. In the studies of Izzo et al. in patients with diagnosed pancreas divisum and ARP, coexisting pathologies in the biliary duct have been found in the MRCP examination. After cholecystectomy ARP symptoms have regressed in all patients during 3 years of follow-up ${ }^{[16]}$. In our study, anomalies in the bile ducts were not revealed in a patient with a confirmed pancreas divisum. There were also no accompanying mutations, which, in particular mutations in the CFTR gene, are suggested as responsible for the development of symptoms of the ARP in patients with pancreas divisum ${ }^{[16]}$. The involvement of many factors in initiating the inflammatory process in the pancreas and its progression to recurrent and chronic pancreatitis seems to be beyond doubt. In the case of single episodes of acute pancreatitis, this issue does not seem so obvious. In the studies performed in our center, the share of the infectious agent was confirmed on the basis of serological tests and clinical symptoms in $40.7 \%$ of patients with AP.

Data presented by Bai et al. have identified infections in $10 \%$ of patients with $\mathrm{AP}^{[1]}$. In the case of single episodes of $\mathrm{AP}$, patients are often treated in regional centers without extensive epidemiological diagnostics, and the data presented in the literature are most often retrospective or come from individual case reports $[17,18,19]$. Also the influence of drugs on the occurrence of pancreatitis is beyond doubt. In our studies, the association of acute pancreatitis with the pharmacotherapy was observed in six children $(22,2 \%)$. In two patients AP occurred in the course of treatment of epilepsy with valproic acid, in one, bceuse of the need to maintain pharmacotherapy, AP has evolved into ARP.

In four others, AP occurred as an effect of azathioprine intake because of inflammatory bowel disease. In these patients no additional triggering factors were established, however, the relationship between the onset of AP and autoimmune disease should be taken into consideration. This result was not a surprise to 
us, because valproic acid is administered as the drug that most often causes acute pancreatitis, and the participation of azathioprine in inducing AP is also common ${ }^{[20,21,22]}$. The increase in the incidence of autoimmune diseases, including inflammatory bowel diseases, increases the common use of the drug and consequently, the increase in the occurrence of adverse reactions. During the study, we observed elevation of pancreatic enzymes during administration of azathioprine in three consecutive children (two-fold increase), however they failed to meet the criteria for diagnosis of AP, i.e. a three-fold increase in amylase and / or lipase, typical clinical symptoms, changes in pancreatic imaging, therefore, they were not included in the study groups. However, the topic requires continuation of studies on the long-term influence of a moderate increase in pancreatic enzymes in the course of treatment of chronic diseases. In summary, the etiological factor can be determined in about $80 \%$ of patients with AP, this percentage depends to a large extent on the reference of the center in which the child is treated. In patients with genetic predisposition, bile and pancreas disorder, chronic diseases, can evolve into acute recurrent pancreatitis and then into chronic pancreatitis. Determining the risk factors seems to be extremely important and influences the patient's follow-up, in certain cases it enables the removal of the triggering factor (obstructive), it also allows to modify the lifestyle of the child and family reducing the risk of relapses of acute recurrent pancreatitis.

\section{Financial support}

None

\section{Potential competing interests}

None

\section{References}

[1] Bai HX, Lowe ME, Husian SZ. What have we learned about acute pancreatitits in children? J Pediatr Gastroenterol Nutr. 2011 Mar;52(3):262-70.

[2] Oracz G, Wejnarska K, Kolodziejczyk E, Kierkus J. Pediatric Acute and Chronic Pancreatitis: Increase in Incidence or Increasing Awareness? Pancreas. 2017 Jul;46(6)

[3] Morinville VD, Husain SZ, Bai H, Barth B, Alhosh R, Durie PR, et al. INSPPIRE Group. Definitions of pediatric pancreatitis and survey of present clinical practices. J Pediatr Gastroenterol Nutr. 2012 Sep;55(3):261-5.

[4] Gariepy CE, Heyman MB, Lowe ME, Pohl JF, Werlin SL, Wilschanski M, et al. Causal Evaluation of Acute Recurrent and Chronic Pancreatitis in Children: Consensus From the INSPPIRE Group. J Pediatr Gastroenterol Nutr. 2017 Jan;64(1):95-103.

[5] Abu-El-Haija M, Kumar S, Szabo F, et al.; NASPGHAN Pancreas Committee. Classification of Acute Pancreatitis in the Pediatric Population: Clinical Report From the NASPGHAN Pancreas Committee. J Pediatr Gastroenterol Nutr. 2017 Jun;64(6):984-990.

[6] Abu-El-Haija M, Kumar S, Quiros JA, et al. The Management of Acute Pancreatitis in the Pediatric Population: A Clinical Report from the NASPGHAN Pancreas Committee. J Pediatr Gastroenterol Nutr. 2017 Aug 23 .
[7] Giefer MJ, Lowe ME, Werlin SL, et al. Early-Onset Acute Recurrent and Chronic Pancreatitis Is Associated with PRSS1 or CTRC Gene Mutations. J Pediatr. 2017 Jul.

[8] Hegyi E, Sahin-Tóth M. Genetic Risk in Chronic Pancreatitis: The Trypsin-Dependent Pathway. Dig Dis Sci. 2017 Jul;62(7).

[9] Kumar S, Ooi CY, Werlin S, et al. Risk Factors Associated With Pediatric Acute Recurrent and Chronic Pancreatitis: Lessons From INSPPIRE. JAMA Pediatr. 2016 Jun $1 ; 170(6)$

[10] Schwarzenberg SJ, Bellin M, Husain SZ, et al. Pediatric chronic pancreatitis is associated with genetic risk factors and substantial disease burden. J Pediatr. 2015 Apr;166(4).

[11] Rosendahl J, Landt O, Bernadova J, et al. CFTR, SPINK1, CTRC and PRSS1 variants in chronic pancreatitis: is the role of mutated CFTR overestimated? Gut. 2013 Apr;62(4)

[12] Chen JM, Férec C. Chronic pancreatitis: genetics and pathogenesis. Annu Rev Genomics Hum Genet. 2009;10

[13] Schneider A, Larusch J, Sun X, et al. Combined bicarbonate conductance-impairing variants in CFTR and SPINK1 variants are associated with chronic pancreatitis in patients without cystic fibrosis. Gastroenterology. 2011 Jan;140(1)

[14] Sánchez-Ramírez CA, Larrosa-Haro A, Flores-Martínez S, Sánchez-Corona J, Villa-Gómez A, Macías-Rosales R. Acute and recurrent pancreatitis in children: etiological factors. Acta Paediatr. 2007 Apr;96(4)

[15] Troendle DM, Fishman DS, Barth BA, et al. Therapeutic Endoscopic Retrograde Cholangiopancreatography in Pediatric Patients With Acute Recurrent and Chronic Pancreatitis: Data From the INSPPIRE (INternational Study group of Pediatric Pancreatitis: In search for a cuRE) Study. Pancreas. 2017 Jul;46(6)

[16] Dumonceau JM, Delhaye M, Tringali A, et al. Endoscopic treatment of chronic pancreatitis: European Society of Gastrointestinal Endoscopy (ESGE) Clinical Guideline. Endoscopy. 2012 Aug;44(8)

[17] Izzo P, Di Cello P, Pugliese F, et al. Pancreas divisum: correlation between anatomical abnormalities and bile precipitation in the gallbladder in seven patients. G Chir. 2016 Jul-Aug;37(4).

[18] Franco J, Fernandes R, Oliveira M, et al. Acute pancreatitis associated with varicella infection in an immunocompetent child. J Pediatr Child Health. 2009;45

[19] al-Abassi A. Acute pancreatitis associated with Mycoplasma pneumoniae: a case report of missed diagnosis. Med Princ Pract. 2002;11

[20] Torre JA, Martin JJ, Garcia CB, et al. Varicella infection as a cause of acute pancreatitis in an immunocompetent child. Pediatr Infect Dis J. 2000;19

[21] Bai HX, Ma MH, Orabi AI, et al. Novel characterization of drug-associated pancreatitis in children. J Pediatr Gastroenterol Nutr. 2011 Oct;53(4)

[22] Cofini M, Quadrozzi F, Favoriti P, Favoriti M, Cofini G. Valproic acid-induced acute pancreatitis in pediatric age: case series and review of literature. G Chir. 2015 JulAug;36(4)

[23] Jamer $\mathrm{T}$, et al. Etiology of acute pancreatitis underestimated problem in paediatrics. Dev Period Med. 2015 
cc (7) Open Access This article is licensed under a Creative Commons Attribution 4.0 International License, which permits use, sharing, adaptation, distribution and reproduction in any medium or format, as long as you give appropriate credit to the original author(s) and the source, provide a link to the Creative Commons license, and indicate if changes were made. The images or other third party material in this article are included in the article's Creative Commons license, unless indicated otherwise in a credit line to the material. If material is not included in the article's Creative Commons license and your intended use is not permitted by statutory regulation or exceeds the permitted use, you will need to obtain permission directly from the copyright holder. To view a copy of this license, visit https://creativecommons.org/licenses/by/4.0/.

(C) The Author(s) 2021 